\title{
Effects of Centrally Administered Etanercept on Behavior, Microglia, and Astrocytes in Mice Following a Peripheral Immune Challenge
}

\author{
Marie lou Camara',2, Frances Corrigan ${ }^{3}$, Emily J Jaehne', Magdalene C Jawahar', Helen Anscomb ${ }^{2}$ and \\ Bernhard T Baune*,
}

'Discipline of Psychiatry, School of Medicine, University of Adelaide, Adelaide, SA, Australia; '2Discipline of Anatomy, School of Medicine, James Cook University, Townsville QLD, Australia; ${ }^{3}$ Discipline of Anatomy and Pathology, School of Medical Sciences, University of Adelaide, SA, Australia

\begin{abstract}
Peripheral cytokines affect central nervous system (CNS) function, manifesting in symptoms of anxiety and cognitive decline. Although the peripheral blockage of tumor necrosis factor (TNF)- $\alpha$ has been effective in alleviating depression and rheumatoid arthritis, it is yet unknown whether central blockade of TNF- $\alpha$ is beneficial for immune-challenged CNS function. This study investigated the effects of central etanercept administration following a peripheral immune challenge on anxiety-like and cognition-like behaviors and microglia and astrocyte numbers. Twelve-week-old C57BL/6 mice $(n=40)$ were treated with either LPS or saline administered peripherally $24 \mathrm{~h}$ before being treated with either etanercept or artificial CSF (aCSF) by intracerebroventricular injection. Mice underwent behavioral analyses for locomotion, memory, and anxiety-like behavior $24 \mathrm{~h}$ post-etanercept/aCSF treatment, and tissue was collected to estimate the numbers of hippocampal microglia and astrocytes. Following peripheral immune challenge with LPS, mice showed increased anxietylike behavior, which was significantly improved following treatment with etanercept (two-way ANOVA: Interaction: $F_{(I, 30)}=0.60$, $P=0.44$; Saline/LPS challenge: $F_{(1,30)}=23.92, P<0.0001$, etanercept vs aCSF: $\left.F_{(1,30)}=11.09, P=0.0023\right)$. For cognition, a significant interaction effect found by two-way ANOVA (Interaction: $F_{(1,20)}=4.96, P=0.037$, Saline/LPS challenge: $F_{(1,20)}=4.966, P=0.31$, aCSF/ etanercept treatment: $\left.F_{(1,20)}=0.06, P=0.80\right)$ and post-hoc analysis revealed a significant decrease in cognition in LPS-aCSF compared with Sal-aCSF mice $(P=0.038)$, but no significant difference was noted between LPS-aCSF and LPS-Etan mice $(P>0.9)$. A significant reduction in the number of microglia within the hippocampus of these mice was noted (two-way ANOVA: Interaction: $F_{(1,15)}=\mid I .4 I$, $P=0.0041$; Saline/LPS challenge: $F_{(1,15)}=50.13, P<0.0001$, etanercept vs aCSF: $\left.F_{(1,15)}=3.36, P=0.08\right)$. Centrally administered etanercept improved anxiety-like behavior but not spatial memory under a peripheral immune challenge and was associated with a decrease in the hippocampal microglia numbers. This suggests that etanercept recovers anxiety-like behavior possibly mediated by a reduction of TNF- $\alpha$-related central inflammation.

Neuropsychopharmacology (20I5) 40,502-5 I2; doi:I0.1038/npp.20 I4.199; published online 17 September 20I4
\end{abstract}

\section{INTRODUCTION}

Tumor necrosis factor (TNF)- $\alpha$ has an important role in the development and progression of cognitive decline, as well as depressive and anxiety disorders (Bai et al, 2013; Baune et al, 2012; Camara et al, 2013; Kaster et al, 2012). Indeed, peripheral inflammatory conditions like rheumatoid arthritis (RA) and psoriasis that show high levels of circulating TNF- $\alpha$ are associated with psychiatric symptoms, such as cognitive decline, anxiety, and depression (Bassukas et al, 2008; Chandarana et al, 1987; Menter et al, 2010).

This association is likely related to extensive cross-talk occurring between immune cells within the central nervous

\footnotetext{
* Correspondence: Professor BT Baune, Discipline of Psychiatry, School of Medicine, University of Adelaide, Adelaide, SA 5005 Australia, Tel: + | 6188222 5I4I, Fax: + | 61882222774 ,

E-mail: Bernhard.Baune@Adelaide.edu.au

Received 2 I March 2014; revised 22 July 20I4; accepted 31 July 20।4; accepted article preview online 8 August 2014
}

system (CNS), such as microglia, and peripheral TNF- $\alpha$ producing monocytes (Kerfoot et al, 2006). Peripheral lipopolysaccharide (LPS) inflammation activates Toll-like receptor 4 present on circumventricular organs and choroid plexus, resulting in $\mathrm{NF} \kappa \mathrm{B}$ activation and subsequent production of TNF- $\alpha$. This activates microglia (Rivest, 2003), creating a positive feedback loop whereby activated microglia produce more TNF- $\alpha$ and other pro-inflammatory cytokines (Nadeau and Rivest, 2000). These pro-inflammatory cytokines activate neurons, causing an increase in $\Delta$ FosB within regions such as the limbic system that govern mood-like behaviors (Frenois et al, 2007). Indeed, infiltration of immune cells into the CNS can result in behavioral changes manifested by increased anxiety and depression (Dantzer et al, 2008).

One current method used to abate this inflammatory process is through the use of TNF- $\alpha$ antagonists, such as etanercept. It is a large (molecular weight of $150000 \mathrm{Da}$ ) fusion protein that consists of extracellular region of human TNF-R2 fragment coupled to an Fc region of human IgG 
that binds to transmembrane TNF- $\alpha$ and acts as a decoy receptor (Mohler et al, 1993). Etanercept is clinically used in the treatment of RA and psoriasis, via subcutaneous administration (Hunt and Emery, 2013; Tyring et al, 2007), and reduces co-morbidities of depression and cognitive impairment (Kekow et al, 2011; Tyring et al, 2006).

As this drug cannot cross the blood-brain barrier (BBB) due to its large size (Boado et al, 2010), it is unable to directly interact with TNF- $\alpha$ present within the CNS and exerts its effects peripherally. However, etanercept indirectly reduces CNS inflammation by decreasing peripheral inflammation and preventing cross-talk (Kerfoot et al, 2006). This principal was evident in a 12 week treatment trial in psoriasis patients, where improved depressive symptoms were observed following subcutaneous etanercept injections (Tyring et al, 2006).

However, it is uncertain whether central blockade of TNF- $\alpha$ is efficacious in preventing CNS manifestations of peripheral inflammatory diseases. Furthermore, although research into the effects of centrally and peripherally administered TNF- $\alpha$ blockade on cognition and depressive symptoms has improved our understanding of cytokine actions in the CNS (Couch et al, 2013; Tobinick and Gross, 2008), little work has been undertaken to enhance our knowledge on the effects of TNF- $\alpha$ blockade on anxiety behaviors. This is surprising as peripheral inflammatory conditions, such as RA and psoriasis, involving TNF- $\alpha$ upregulation are commonly associated with anxiety (Chen et al, 2013; Salim et al, 2011), although the exact mechanisms whereby inflammation induces psychiatric symptoms are unclear (Chandarana et al, 1987; Couch et al, 2013). It is suggested that peripheral inflammation can cause upregulation of TNF- $\alpha$, which in turn activates macrophages peripherally and microglia centrally (Qin et al, 2007) and is further linked to the development of anxiety-like behavior (Wohleb et al, 2011). Recently, a study has shown that chronic peripheral administration of etanercept in rats reduced both depression- and anxiety-like behaviors in the absence of immune stimulation (Bayramgurler et al, 2013). Although the study showed that chronic blockade of TNF- $\alpha$ action under physiologically normal conditions may be beneficial against anxiety-like behavior (Bayramgurler et al, 2013), it is unclear whether this mechanism will translate to peripheral inflammatory conditions. Astrocytes are known to be activated by LPS stimulation to produce cytokines such as TNF- $\alpha$, though the involvement in the development of behavioral disruptions is unknown (Zhang et al, 2014).

The present study sought to first determine the effects of peripheral immune challenge on anxiety- and cognition-like behaviors by treating mice with an acute dose of LPS. Due to the link between peripheral cytokines and immune cells of the CNS (Kerfoot et al, 2006), we also sought to understand whether a single peripheral immune challenge would be sufficient to activate CNS immune cells. Our next aim was to investigate whether central blockage of TNF- $\alpha$ by etanercept would reverse the behavioral and neurobiological effects seen after LPS challenge. We hypothesized that centrally administered etanercept reduces anxiety-like behaviors, improves cognition-like behavior, and reduces CNS inflammation in mice that have received a peripheral immune challenge.

\section{MATERIALS AND METHODS}

\section{Mice}

Male C57BL/6 mice (Jackson stock number: 000664), aged 12 weeks, were purchased from the University of Adelaide breeding facility and used in this study $(n=40)$. Male mice were chosen for this study to avoid the possibility of confounding factors arising out of sexually dimorphic behavior (Brown et al, 2000). All mice were housed in groups of five per cage during the experimental period, with food and water available ad libitum. Ambient temperature of the housing and testing rooms was $22 \pm 1{ }^{\circ} \mathrm{C}$. Mice were housed under a 12-h light-dark cycle, lights on at 0700 hours, and all behavioral testing was conducted between 0800 and 1600 hours. Animal procedures were approved by the University of Adelaide Animal Ethics committee.

\section{Drugs}

LPS. To induce a systemic inflammatory reaction for experimental procedures, LPS from Escherichia coli (Sigma Chemical, St Louis, MO; 0111:B4) was diluted in saline and injected via intraperitoneal injection (IP) at a dose of $1 \mathrm{mg} / \mathrm{kg}$ at 1400 hours. Control mice were injected with saline only by IP injection. Pilot studies in our lab using the same dose of LPS showed inflammation $24 \mathrm{~h}$ post-LPS in the form of increased serum levels of the cytokines TNF- $\alpha$, IL- 6 , and MCP-1, as well as hippocampal and prefrontal cortex (PFC) gene expression of TNF- $\alpha$, IL- $1 \beta$ and IL-10. This led us to choose this dose of LPS and time point for administering etanercept, in the hope that it would decrease TNF- $\alpha$ and other signs of inflammation, including glia.

Etanercept. Etanercept (Enbrel, Wyeth-Ayerst Laboratories, $25 \mathrm{mg} / \mathrm{kg}$ ) was diluted $1: 10$ in artificial cerebrospinal fluid (aCSF, composition detailed in Supplementary Table S1) and administered intracerebroventricularly (ICV). A total volume of $2 \mu \mathrm{l}$ was injected as previously established by Nilsberth et al (2009). Eighteen hours post-LPS/saline administration, mice were anesthetized with isoflurane (1\%), mounted in a stereotaxic frame, and kept at $37^{\circ} \mathrm{C}$ through a feedback-controlled heating pad. A $0.3-\mathrm{mm}$ burrhole was made at the point relative to Bregma: $1 \mathrm{~mm}$ to the right and $0.5 \mathrm{~mm}$ posterior to inject into the lateral ventricle. A 33-gauge needle connected to a 5- $\mu$ l Hamilton syringe was then lowered $2.5 \mathrm{~mm}$ and either etanercept or aCSF $(2 \mu \mathrm{l})$ was injected at a rate of $0.5 \mu \mathrm{l} / \mathrm{min}$. The needle was then left in place for $2 \mathrm{~min}$, before being removed to suture the skin. Mice were then placed on a heat pad to recover. Once mice had regained normal mobility, they were returned to their home cage with unlimited access to food and water and checked regularly for $24 \mathrm{~h}$ to ensure that there were no adverse effects from surgery.

\section{Design and Treatment Groups}

Mice were divided into four treatment groups $(n=40)$. Two groups were challenged with an acute dose of LPS $(n=20$; IP administration) while two groups received saline $(n=20$; IP administration). Of the LPS groups, one group received etanercept via ICV administration ( $n=10$; LPS-Etan: LPS challenge IP with etanercept administered ICV) and a 
control group received aCSF also via ICV administration ( $n=10$; LPS-aCSF: LPS challenge IP with aCSF administered ICV). Similarly with the saline-treated mice, one group $(n=10)$ received etanercept through ICV injection (Sal-Etan: saline treatment IP with etanercept administered ICV) while the other group $(n=10)$ received aCSF also through an ICV injection (Sal-aCSF: saline treatment IP with aCSF administered ICV) (Table 1).

\section{Behavioral Analysis}

A behavioral battery was carried out to assess locomotor activity, anxiety-like behavior, and cognition-like behavior with an hour between tests to prevent interference. Equipment for all tests was purchased from Stoelting (USA), and all movements were tracked using the ANYMAZE imaging software Stoelting (USA).

Locomotor activity: open field test (OFT). Basal locomotor activity was measured in the OFT. Mice were placed at the center of a brightly lit plexi-glass box $\left(40 \times 40 \mathrm{~cm}^{2}\right)$, and movements were tracked over a 5 -min period as previously published (Camara et al, 2013). Distance travelled and time spent in the outer and inner zones of the box were measured.

Anxiety-like behavior: elevated zero maze (EZM). The EZM measures anxiety-like behavior and consists of a grey perspex circular platform of $50 \mathrm{~cm}$ diameter and $5 \mathrm{~cm}$ width, at a height of $40 \mathrm{~cm}$. The platform was divided into four equal quadrants (arms), two closed quadrants with grey Perspex walls $(27 \mathrm{~cm}$ high) on the inside and outside, and two open quadrants without walls. The EZM and the elevated plus maze (EPM) provide a similar evaluation of anxiety-like behavior, but the circular design of the EZM eliminates the difficulty in the EPM of interpreting time spent in the central square (Shepherd et al, 1994).

Mice were placed at the center of either one of the open arms and allowed to explore the apparatus for $5 \mathrm{~min}$. Time spent in open arms was used as a measure of anxiety-like behavior, where more anxious mice spend less time in the open arms. Head dipping behavior and time spent in stretch/attend posture from the closed arm were also recorded as measures of anxiety-like behavior wherein increased time spent in these behaviors was taken as a measure of low anxiety-like response.

Spatial recognition memory: Y Maze. The Y maze assesses hippocampal spatial recognition memory and consists of a three-armed chamber, with the arms at a $120^{\circ}$ angle from each other. Each arm is $35 \mathrm{~cm}$ long, $5.0 \mathrm{~cm}$ wide, and $10 \mathrm{~cm}$ high (Choy et al, 2008).

Phase 1. One arm of the maze was closed off, and mice were placed at the bottom of the 'start' arm and allowed to explore the two arms for $10 \mathrm{~min}$.

Phase 2. Forty-five minutes after phase 1mice were tested with all three arms left open. Mice were again placed at the bottom of the start arm and allowed to explore the three arms for $5 \mathrm{~min}$. As mice have a preference for exploring novel environments, normal mice should spend more time exploring the novel arm (Dulawa et al, 1999). A preference for the novel arm for each mouse was calculated as follows: Time in novel arm/(Time in novel arm + time in familiar arm $) \times 100$. Mice that did not enter either the familiar or novel arms during Phase 2 were excluded from the analysis.

\section{Gene Expression Analysis (RT-qPCR)}

A subset of mice was selected for qPCR analysis to measure the expression of Tnfa post-LPS and etanercept treatment ( $n=5 /$ treatment group). Mice were killed via a lethal dose of pentobarbital via IP injection, and the hippocampal and PFC tissue from mice was collected and stored in RNAlater (Ambion, Life Technologies) at $-20^{\circ} \mathrm{C}$ until required for RNA extraction. The hippocampus was analyzed as it has a role in spatial cognition (Sweatt, 2004) as well as some emotional responses (Sweatt, 2004), while the PFC was chosen for analysis as it has a role in cognitive control (Miller and Cohen, 2001) and goal-oriented emotional processing (Davidson, 2002).

Brain tissue was lysed with TissuelyserLT (Qiagen, Australia) with $5-\mathrm{mm}$ stainless steel lysis bead (Qiagen). RNA was then extracted from the lysed tissue using PureLink RNA mini extraction kit (Ambion) as per the manufacturer's instructions. RNA was then quantified using Nanodrop Lite (Thermo Fischer Scientific). Gene expression analysis for Tnfa was performed by RT-qPCR in two steps. First, complementary DNA (cDNA) was synthesized from the total RNA using the SuperScript III First-Strand Synthesis System (Invitrogen) in a thermal cycler (MJ Research) following the manufacturer's instructions. The products were then diluted to attain uniform concentration of $10 \mathrm{ng} / \mu \mathrm{l}$ of cDNA for qPCR.

In the next step, qPCR was performed on the cDNA using the Applied Biosystems 7500 Fast real-time PCR machine

Table I Dosing and Testing Schedule for Experiments

\begin{tabular}{|c|c|c|c|}
\hline Day I & Day 2 & Day 3 & Day 4 \\
\hline \multirow[t]{3}{*}{$\begin{array}{l}\text { Saline dosing via IP injection }(n=10) \\
(1300 \text { hours })\end{array}$} & $\begin{array}{l}\text { aCSF treatment by ICV injection }(n=10) \\
\text { (Start at } 0900 \text { hours) }\end{array}$ & $\begin{array}{l}\text { Etanercept treatment by } \\
\text { ICV injection }(n=10)\end{array}$ & OFT (0900-1000 hours) \\
\hline & $\begin{array}{l}\text { LPS dosing via IP injection }(n=10) \\
\text { (1300 hours })\end{array}$ & & Y maze (I300-I500 hours) \\
\hline & & & Tissue collection \\
\hline
\end{tabular}

The experimental set-up was conducted over 2 weeks with $n=20$ animals treated and tested per week.

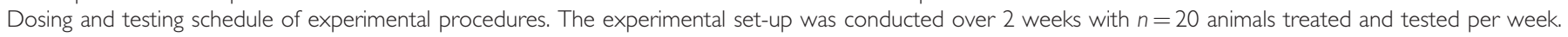


(ABI, Life technologies). Expression of Tnfa was quantified with Gapdh used as the endogenous reference gene using the primers listed below;

Tnfa-forward: 5'-CCACCACGCTCTTCTGTCTA-3';

Tnfa-reverse: $5^{\prime}$-AGGGTCTGGGCCATAGAACT-3';

Gapdh-forward: 5'-TGTTCCTACCCCCAATGTGT-3'; and

Gapdh-reverse: 5'-CCTGCTTCACCACCTTCTTG-3'.

Samples and negative controls (without cDNA) for each gene were run in triplicates on a 96-well MicroAmp Fast optical-qPCR plate (ABI, Life technologies). Briefly, $4 \mu \mathrm{l}$ of cDNA was added to a master mix consisting of $10 \mu$ of $2 \mathrm{X}$ Power SYBR Green PCR master mix (ABI, Life technologies), and $0.14 \mu \mathrm{l}$ each of forward and reverse primer along with $5.72 \mu \mathrm{l}$ of double distilled water to make a total reaction volume of $20 \mu \mathrm{l}$. The raw data consisting of the Ct (Cycle threshold) values for individual reactions were exported to excel files, and analysis was conducted manually using the delta-delta CT method (Livak and Schmittgen, 2001).

\section{Immunohistochemistry (IHC)}

Following behavioral testing, animals were injected IP with pentobarbital and perfused via transcardiac injection with $10 \%$ neutral buffered formalin. Brains were embedded in paraffin wax, and $5 \mu \mathrm{m}$ sections spaced $150-\mu \mathrm{m}$ apart of the hippocampus and PFC were taken. After de-waxing and dehydrating slides, endogenous peroxidases were blocked, before antigen retrieval in citrate solution. Non-specific binding was then blocked with normal horse serum, before application of primary antibody with incubation overnight (Abcam, IBA1 1:1000, Santa Cruz, GFAP 1:40000, Dako). The next day, slides were incubated with the appropriate species of secondary antibody (Abacus, 1:250) followed by streptavidin peroxidase (list of antibodies detailed in Supplementary Table S2). The antigen-antibody complex was then detected with diaminobenzadine Sigma) followed by counter staining with hematoxylin prior to dehydration and mounting with DePex mounting media.

Slides were digitally scanned using the Nanozoomer (Hamamatsu) and viewed with the associated software (NDP view). Serial images of the dentate gyrus of each section were captured as jpegs and then exported to Image J (NIH) to count number of positive cells.

\section{Statistical Analysis}

Data analyses were carried out using the GraphPad Prism software (version 6.01). All data are presented as mean \pm SEM. Comparisons between groups were carried out using two-way ANOVA with Bonferroni's multiple comparisons post-hoc test where appropriate. Weight of mice was analyzed using a $2 \times 2 \times 3$ ANOVA. A significance level of $P<0.05$ was regarded as significant in all tests.

\section{RESULTS}

\section{Age and Weight of Animals}

All male mice that were used in this study were tested and killed at 13 weeks of age. Weight was measured at baseline and $24 \mathrm{~h}$ following LPS challenge as well as $24 \mathrm{~h}$ following etanercept treatment ( $48 \mathrm{~h}$ after LPS). Change in weight over time was analyzed using a $2 \times 2 \times 3$ ANOVA. Tests of within-subjects' measures showed a significant effect of time $\left(\mathrm{F}_{(2,36)}=42.2, P<0.001\right)$, indicating a decrease in weights over the 48 -h period of immune challenge and etanercept treatment. In addition, a significant effect of time $\times$ LPS $\left(\mathrm{F}_{(2,36)}=23.6, \quad P<0.001\right)$ was observed, indicating LPS significantly reduced weights in mice over time. However, an effect of time $\times$ etanercept was not seen $\left(\mathrm{F}_{(2,36)}=2.34\right.$, $P=0.10$ ), suggesting that etanercept had no effect on weight and was not able to reverse the decrease in weight caused by LPS treatment. No significant three-way interaction between time, LPS, and etanercept $\left(\mathrm{F}_{(2,36)}=0.99, P=0.37\right)$ was observed (Figure 1).

\section{Locomotor Activity}

Locomotor activity was measured under stressful conditions using the open field test. Analysis by two-way ANOVA found no interaction effect or effect of etanercept treatment on locomotor activity, but a significant effect of LPS challenge was observed (Figure 2: Two-way ANOVA: Interaction: $\mathrm{F}_{(1,36)}=0.049, P=0.8254$, Saline/LPS challenge: $\mathrm{F}_{(1,36)}=25.84, \quad P<0.0001, \quad$ aCSF/etanercept treatment: $\mathrm{F}_{(1,36)}=1.35$. $\left.P=0.25\right)$. Bonferroni's multiple comparison showed that LPS-aCSF-treated mice were less active than Sal-aCSF-treated mice $(P=0.009)$. These results suggest that, while treatment with LPS impaired locomotor activity, etanercept was ineffective in reversing this impairment.

\section{Anxiety-like Behavior}

Anxiety-like behavior was measured using the EZM (Figure 3). Mice were allowed to move freely between open and closed arms. Time spent in the open arm is regarded as an indicator of lower levels of anxiety-like behavior, as are time spent in head dipping and stretched posture (Shepherd et al, 1994). No significant interaction effect was seen between groups for time in open arms, but a significant

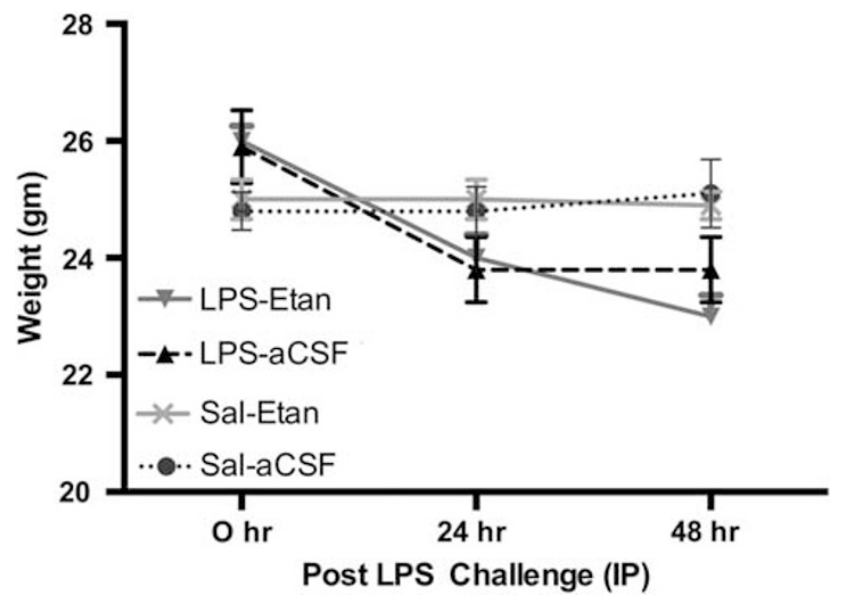

Figure I Weight of mice before and after LPS dosing. The figure depicts the weight of mice over the 48-h experimental period. All data represent mean \pm SEM ( $n=10$ /group). Sal-aCSF: saline treatment IP with aCSF administered ICV, Sal-Etan: saline treatment IP with etanercept administered ICV, LPS-aCSF: LPS challenge IP with aCSF administered ICV, LPSEtan: LPS challenge IP with etanercept administered ICV. Data compared using $2 \times 2 \times 3$-way ANOVA with Bonferroni's multiple comparison test. 
effect for aCSF/etanercept treatment was noted (two-way ANOVA: Interaction: $\mathrm{F}_{(1,31)}=0.64, P=0.42$, Saline/LPS challenge: $\mathrm{F}_{(1,31)}=1.18, P=0.28$, aCSF/etanercept treatment: $\left.\mathrm{F}_{(1,31)}=4.58, P=0.04\right)$.

No interaction effect for time spent in head dipping behavior was observed; however, an effect of LPS challenge and etanercept treatment was seen (two-way ANOVA: Interaction: $\mathrm{F}_{(1,30)}=0.60, P=0.44$, Saline/LPS challenge: $\mathrm{F}_{(1,30)}=23.92, \quad P<0.001, \quad$ aCSF/etanercept treatment: $\mathrm{F}_{(1,30)}=11.09, P=0.0023$; Figure $\left.3 \mathrm{~b}\right)$. Bonferroni's multiple comparison of LPS effect showed that LPS-aCSF-treated mice spent less time in head dipping behavior than Sal-aCSF mice $(P=0.0031)$, suggesting that LPS increases anxietylike behavior. Bonferroni's multiple comparison for etanercept effect showed that LPS-Etan-treated mice spent more time in head dipping behavior than LPS-aCSF mice $(P=0.025)$, indicating that etanercept was able to reverse the effects on LPS on head dipping behavior.

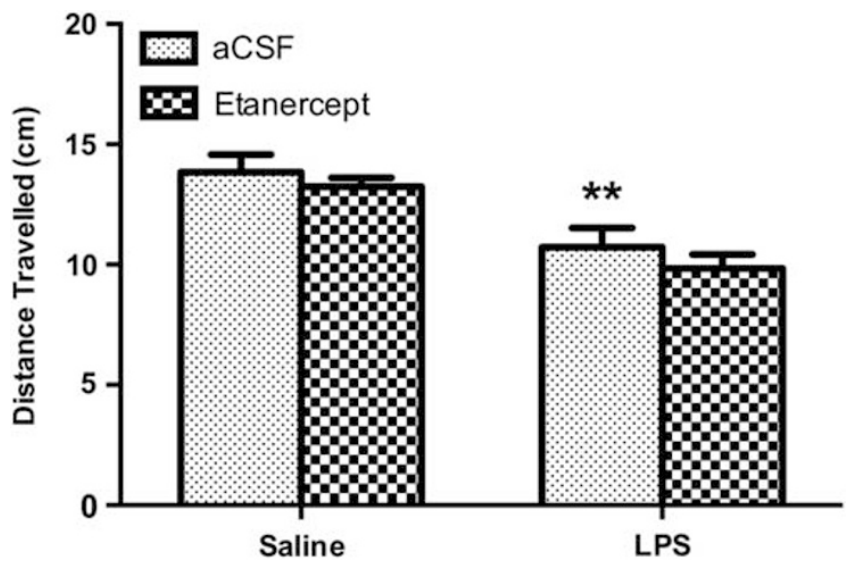

Figure 2 Locomotor activity (OFT). Distance travelled in OFT, as a measure of baseline locomotor activity All data represent mean \pm SEM ( $n=10$ /group). Sal-aCSF: saline treatment IP with aCSF administered ICV, Sal-Etan: saline treatment IP with etanercept administered ICV, LPS-aCSF: LPS challenge IP with aCSF administered ICV, LPS-Etan: LPS challenge IP with etanercept administered ICV. Data compared using two-way ANOVA (Interaction: $P=0.8254$, Saline/LPS challenge: $P<0.001$, aCSF/etanercept treatment: $P=0.25)$. $* * P<0.0$ I LPS-aCSF compared with Sal-aCSF-treated mice, analyzed by Bonferroni's multiple comparison.
Similar results were also seen for stretch-attend posture (two-way ANOVA: Interaction: $\mathrm{F}_{(1,31)}=2.98, P=0.096$; Saline/LPS challenge: $\mathrm{F}_{(1,31)}=14.55, P=0.0006$; aCSF/etanercept treatment: $F_{(1,31)}=13.93, P=0.0008$; Figure $3 c$ ). Bonferroni's multiple comparison of LPS effect showed that LSP-aCSF-treated mice spent less time in stretch-attend posture compared with Sal-aCSF mice $(P=0.0032)$, indicative of LPS-induced anxiety-like behavior. Again, Bonferroni's multiple comparison for etanercept effects showed that LPS-Etan-treated mice spent more time in stretch-attend posture than LPS-aCSF mice $(P=0.002)$, indicating that etanercept can reduce anxiety behaviors in LPS-treated mice.

Distance travelled in the open arm was also measured between groups, and no significant differences were detected (two-way ANOVA: Interaction: $\mathrm{F}_{(1,34)}=1.17, P=0.28$; Saline/LPS challenge: $\mathrm{F}_{(1,34)}=0.15, P=0.69$; aCSF/etanercept treatment: $F_{(1,34)}=1.20, P=0.81$; Mean \pm SEM: SalaCSF: $10.4 \pm 1.05$, LPS-aCSF $8.189 \pm 1.9$, Sal-Etan: $8.45 \pm$ 1.06, LPS-Etan: $9.45+1.83$ ). This indicates that though there were significant changes observed in the OFT between LPS and Saline-treated mice, this difference did not extend to the EZM and the differences observed in this test were reflections of anxiety-like behavior and not locomotor deficits

\section{Cognition-Like Behavior}

The Y maze was used as a measure of spatial recognition memory. A preference index for the novel arm was calculated and a significant interaction effect was found by two-way ANOVA (Interaction: $\mathrm{F}_{(1,20)}=4.96, P=0.037$, Saline/LPS challenge: $\mathrm{F}_{(1,20)}=1.04, P=0.31$, aCSF/etanercept treatment: $\left.\mathrm{F}_{(1,20)}=0.06, P=0.80\right)$. Bonferroni's posthoc analysis revealed a significant decrease in spatial recognition memory in LPS-aCSF compared with Sal-aCSF mice $(P=0.038)$, but no significant difference was noted between LPS-aCSF and LPS-Etan mice $(P>0.9)$, suggesting that this impairment was not rescued by etanercept.

Distance travelled in the Y maze was also measured between groups, and no significant differences were detected (twoway ANOVA: Interaction: $\mathrm{F}_{(1,36)}=0.96, P=0.33$; Saline/LPS

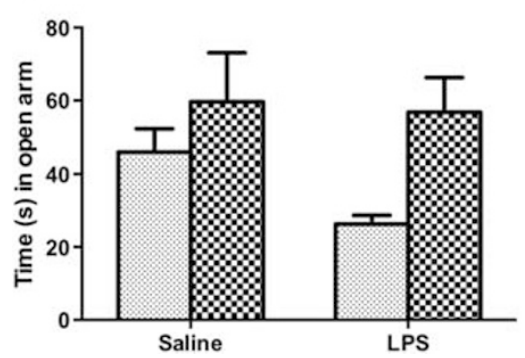

b

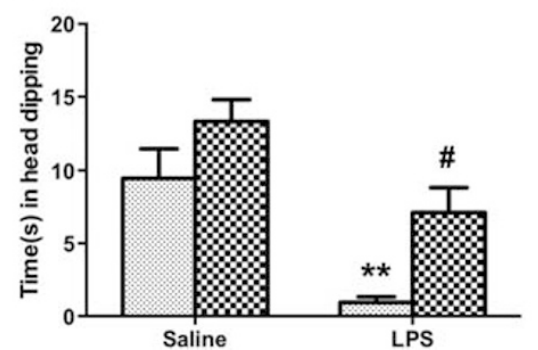

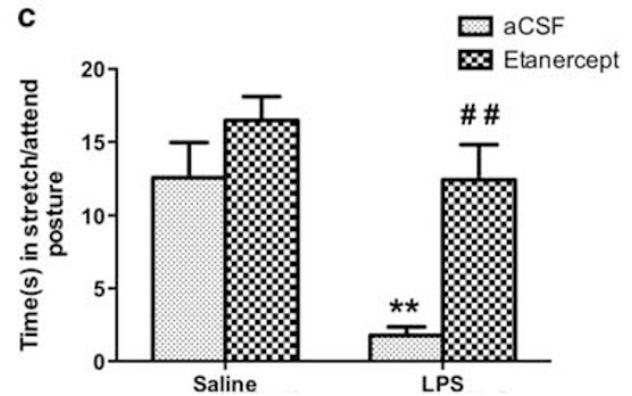

Figure 3 Anxiety-like behavior (EZM). (a) Time spent in open arm, (b) Time spent in head dipping behavior, (c) Time spent in stretch/attend posture. All data represent mean \pm SEM ( $n=$ IO/group). Sal-aCSF: saline treatment IP with aCSF administered ICV, Sal-Etan: saline treatment IP with etanercept administered ICV, LPS-aCSF: LPS challenge IP with aCSF administered ICV, LPS-Etan: LPS challenge IP with etanercept administered ICV. Data compared using two-way ANOVA; ((a) Interaction: $P=0.42$, Saline/LPS challenge: $P=0.28$, aCSF/etanercept treatment: $P=0.04$; (b) Time in head dipping behavior: Interaction: $P=0.44$, Saline/LPS challenge: $P<0.001$, aCSF/etanercept treatment: $P=0.0023$; (c) Stretch-attend posture: Interaction: $P=0.096$, Saline/LPS challenge: $P=0.0006$, aCSF/etanercept treatment: $P=0.0008$ ). ${ }^{*} * P<0.01$ LSP-aCSF compared with Sal-aCSF mice, ${ }^{\#} P<0.05$, ${ }^{\#} P<0.01$ LPS-Etan compared with LPS-aCSF mice, analyzed by Bonferroni's multiple comparisons. 
challenge: $\mathrm{F}_{(1,36)}=0.91, \quad P=0.34 ;$ aCSF/etanercept treatment: $\quad \mathrm{F}_{(1,34)}=1.81, \quad P=0.18$; Mean \pm SEM: Sal-aCSF: $6.39 \pm 1.28$, LPS-aCSF $10.87 \pm 3.78$, Sal-Etan: $5.54 \pm 0.63$, LPS-Etan: $5.47+2.24$ ) (Figure 4 ).

\section{Expression of (mRNA) Tnfa in the Hippocampus and PFC}

Levels of (mRNA) Tnfa in the hippocampus and PFC of mice were measured $48 \mathrm{~h}$ after LPS challenge. This was done first to test whether LPS would increase Tnfa levels in these CNS areas and then to test whether etanercept would be effective in reversing the upregulation of Tnfa.

In the hippocampus (Figure 5a), we found significant difference in mRNA levels of Tnfa between groups (Interaction:

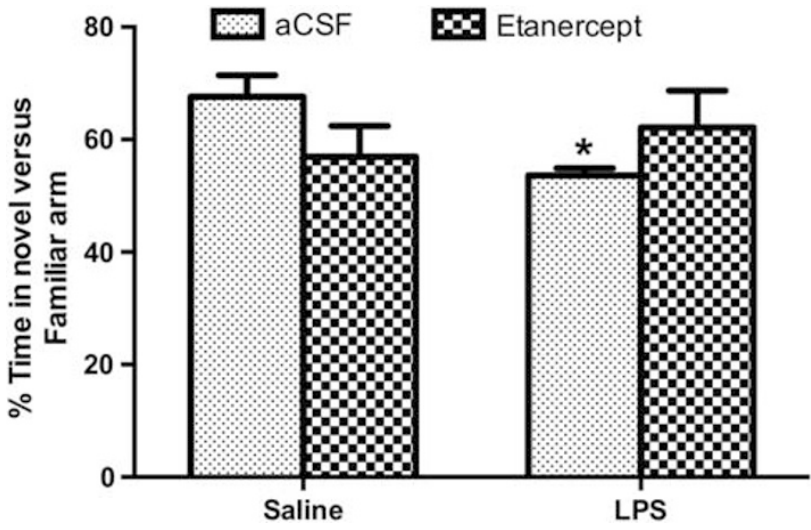

Figure 4 Cognition-like behavior ( $Y$ maze). (a) Percentage of time spent in the novel arm vs the familiar arm of the retention phase of $Y$ maze, All data represent mean \pm SEM ( $n=5-7 /$ group). Sal-aCSF: saline treatment IP with aCSF administered ICV, Sal-Etan: saline treatment IP with etanercept administered ICV, LPS-aCSF: LPS challenge IP with aCSF administered ICV, LPS-Etan: LPS challenge IP with etanercept administered ICV. Data compared using two-way ANOVA (Interaction: $P=0.037$, Saline/LPS challenge: $P=0.31$, aCSF/etanercept treatment: $P=0.80$ ). ${ }^{*} P<0.05$ LPSaCSF compared with Sal-aCSF-treated mice, analyzed by Bonferroni's multiple comparison.
$\mathrm{F}_{(1,13)}=28.04, P=0.0001$, Saline/LPS challenge: $\mathrm{F}_{(1,13)}=$ 24.52, $P=0.0003$, aCSF/etanercept treatment: $\mathrm{F}_{(1,13)}=6.84$, $P=0.021)$. Post-hoc analysis showed that indeed LPS-aCSF mice had significantly higher levels of Tnfa compared with Sal-aCSF mice $(P<0.001)$, indicating that peripheral LPS was effective in increasing CNS Tnfa levels. Furthermore, etanercept treatment was successful in significantly reducing Tnfa levels in LPS-Etan mice compared with LPS-aCSF mice $(P=0.001)$.

In the PFC (Figure 5b), the difference between treatment groups was not as robust as in the hippocampus. Two-way ANOVA comparing the different treatment groups found a significant positive effect of LPS in increasing the Tnfa expression (Interaction: $\mathrm{F}_{(1,15)}=0.09, P=0.76$, Saline/LPS challenge: $\mathrm{F}_{(1,15)}=14.25, P=0.018$, aCSF/etanercept treatment: $\left.\mathrm{F}_{(1,15)}=0.07, P=0.79\right)$ but no significant interaction effects, suggesting that LPS was effective in increasing Tnfa but etanercept was ineffective in reversing this effect. Posthoc analysis of LPS effect confirmed that LPS was effective in increasing the Tnfa levels in LPS-challenged mice (LPSaCSF) compared with Sal-aCSF mice $(P=0.026)$. LPS-Etan mice also had significantly higher Tnfa levels than Sal-Etan mice $(P=0.045)$.

\section{Examination of Microglial and Astrocyte Numbers following LPS Treatment}

To study the effects of LPS and etanercept on resident immune cells in the brain, microglial (IBA1) and astrocyte (GFAP) numbers were examined by staining hippocampal slices using IHC and counting the number of positive cells in the dentate gyrus and the PFC. Immune challenge with LPS had no effect on astrocyte number within the dentate gyrus (two-way ANOVA: Interaction: $\mathrm{F}_{(1,15)}=1.24, P=0.28$, Saline/LPS challenge: $\mathrm{F}_{(1,15)}=0.077, P=0.78$, aCSF/etanercept treatment: $\mathrm{F}_{(1,15)}=3.58, \quad P=0.07$, Supplementary Figure S1A) or in the PFC (two-way ANOVA: Interaction: $\mathrm{F}_{(1,12)}=0.11, P=0.73$, Saline/LPS challenge: $\mathrm{F}_{(1,12)}=0.69$, $P=0.42$ aCSF/etanercept treatment: $\mathrm{F}_{(1,12)}=0.63, P=0.44$, Supplementary Figure S1B).
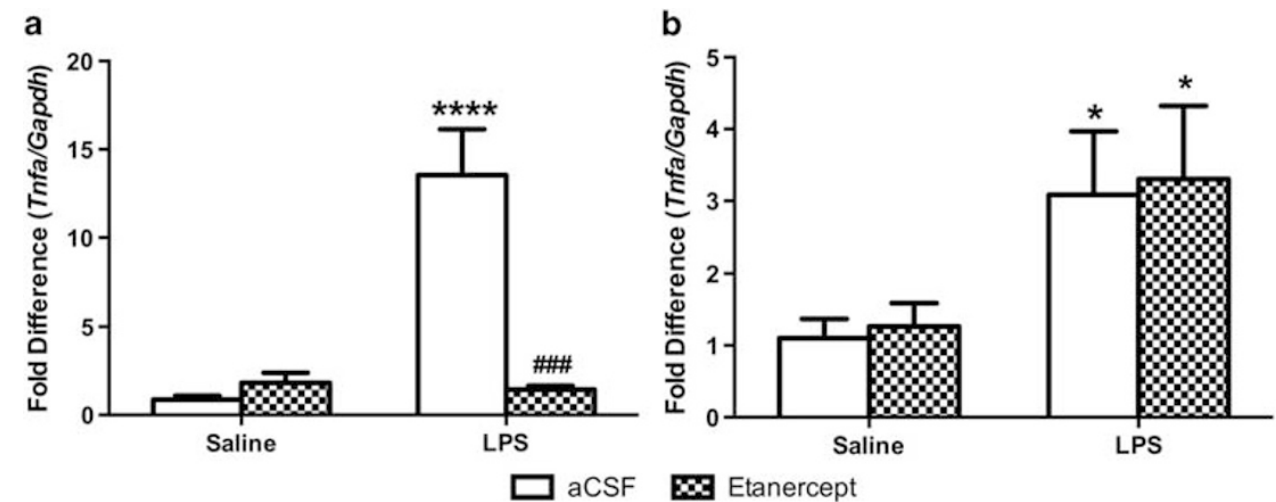

Figure 5 Expression levels of (mRNA) Tnfa in the PFC and hippocampus. (a) Fold difference of Tnfa expression between treatment groups in the hippocampus, (b) fold difference of the Tnfa expression between treatment groups in the PFC. All statistical analysis were done using the delta Ct values (expression of Tnfa normalized to Gapdh), and the data represent mean \pm SEM ( $n=5 /$ group). (Sal-aCSF: saline treatment IP with aCSF administered ICV, Sal-Etan: saline treatment IP with etanercept administered ICV, LPS-aCSF: LPS challenge IP with aCSF administered ICV, LPS-Etan: LPS challenge IP with etanercept administered ICV). Data compared using two-way ANOVA ((a) Interaction: $P=0.000$ I, Saline/LPS challenge: $P=0.0003$, aCSF/etanercept treatment: $P=0.02$ I; (b) Interaction: $P=0.76$, Saline/LPS challenge: $P=0.018$, aCSF/etanercept treatment: $P=0.79$ ). $* P<0.05$, $* * * * P<0.000$ I compared

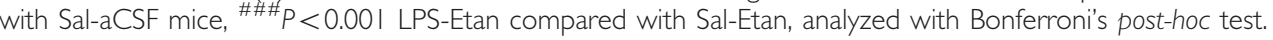


a

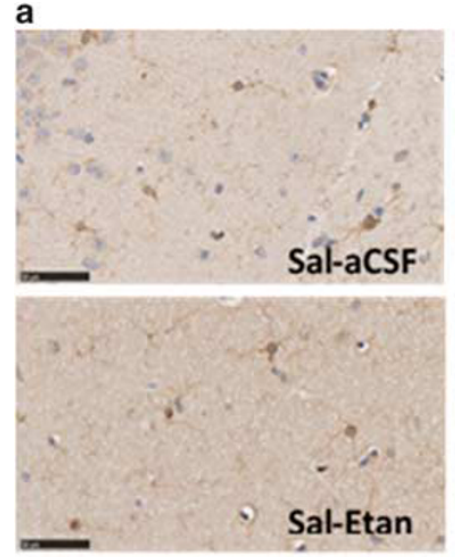

b
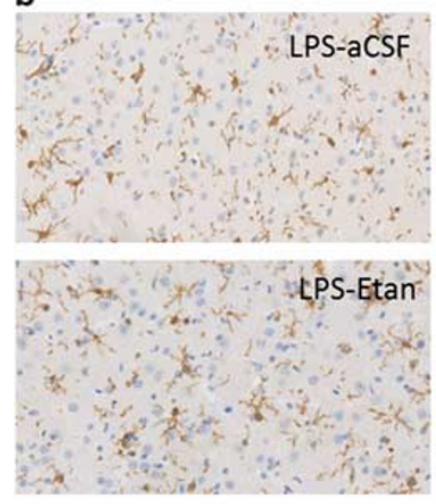
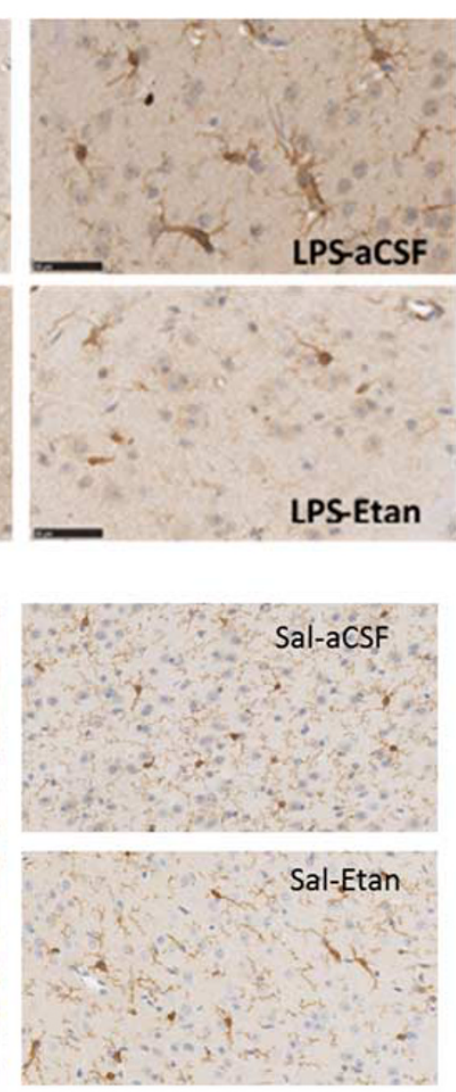
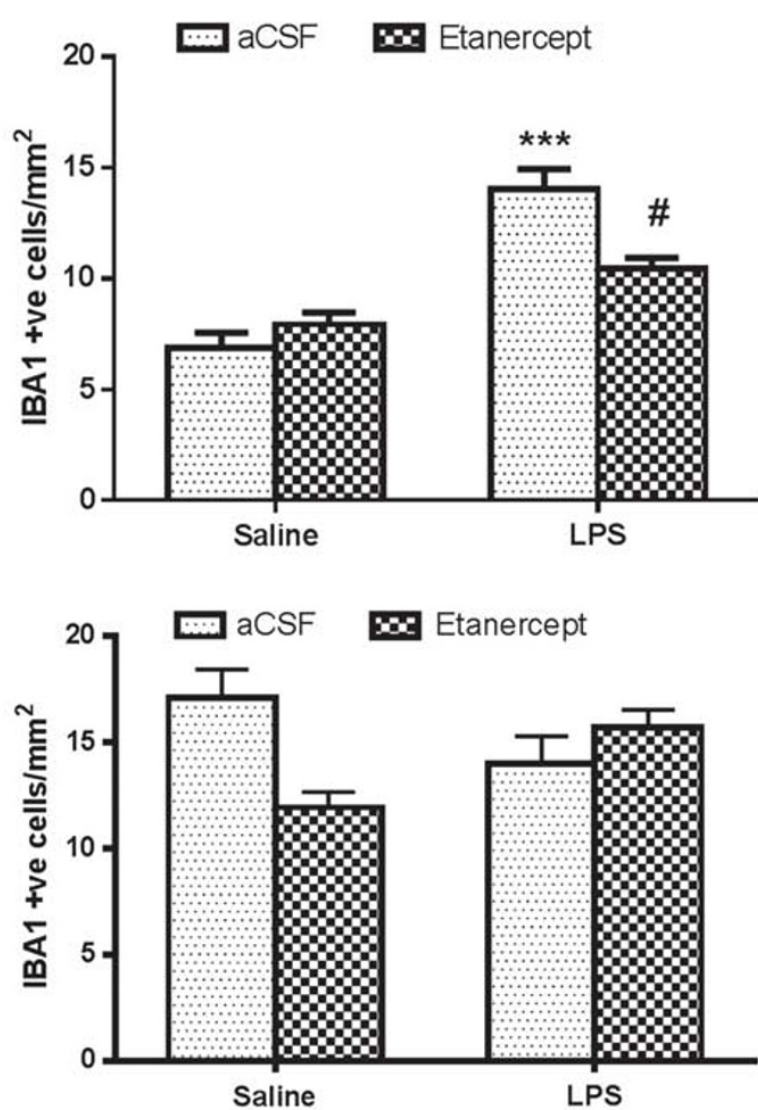

Figure 6 Microglia counts across experimental conditions. (a) The figure shows IHC images of the microglia in the dentate gyrus while the graph represents the number of IBAI-positive cells in the dentate gyrus. (b) The figure shows IHC images of the microglia in the PFC while the graph represents the number of IBAI-positive cells in the PFC. All data represent mean \pm SEM ( $n=5 / g r o u p$, Sal-aCSF: saline treatment IP with aCSF administered ICV, SalEtan: saline treatment IP with etanercept administered ICV, LPS-aCSF: LPS challenge IP with aCSF administered ICV, LPS-Etan: LPS challenge IP with etanercept administered ICV). Data compared using two-way ANOVA ((a) Interaction: $P=0.004 \mathrm{I}$, Saline/LPS challenge: $P<0.00 \mathrm{I}$, aCSF/etanercept: $P=0.08$; (b) Interaction: $P=0.02$, Saline/LPS challenge: $P=0.81$, aCSF/etanercept treatment: $P=0.22$ ). ***** $P<0.00$ I LPS-aCSF compared with Sal-aCSF,

${ }^{\#} P<0.05$ LPS-Etan compared with LPS-aCSF, analyzed by Bonferroni's multiple comparisons.

LPS, however, did lead to a significant increase in microglial numbers in the hippocampus, with a significant interaction effect between LPS challenge and etanercept treatment (two-way ANOVA: Interaction: $\mathrm{F}_{(1,15)}=11.41$, $P=0.0041$, Saline/LPS challenge: $\mathrm{F}_{(1,15)}=50.13, P<0.001$, aCSF/etanercept: $\left.\mathrm{F}_{(1,15)}=3.36, P=0.08\right)$. Bonferroni's multiple comparison of LPS effect on microglial numbers showed that LPS-aCSF-treated mice had higher number of IBA1-positive cells in the dentate gyrus compared with SalaCSF mice $(P<0.001)$. Bonferroni's multiple comparison of etanercept effects showed that LPS-Etan mice had lower number of BA1-positive cells within the dentate gyrus compared with LPS-aCSF $(P=0.016)$. In the PFC, however, we found that, while there was a significant interaction effect between treatment groups, there was no significant effect of LPS or etanercept (two-way ANOVA: Interaction: $\mathrm{F}_{(1,9)}=6.81, P=0.02$, Saline/LPS challenge: $\mathrm{F}_{(1,9)}=0.05$, $P=0.81$, aCSF/etanercept treatment: $\left.\mathrm{F}_{(1,15)}=1.7, P=0.22\right)$. These results indicate that LPS increased microglial numbers only in the hippocampus and not in the PFC and that this increase was prevented with etanercept treatment (Figure 6).

\section{DISCUSSION}

In the present study, we have shown that acute systemic immune challenge with LPS resulted in sickness behavior in mice, evident by a significant decrease in weight at $24 \mathrm{~h}$, and impaired locomotor activity in the OFT. Furthermore, LPS significantly increased some aspects of anxiety-like behavior in the EZM and significantly reduced preference for the novel arm in the Y Maze. Etanercept treatment post-LPS was able to prevent the effects of LPS on anxiety-like behaviors in the EZM, but we were unable to observe a significant effect on cognition-like behavior (Y Maze), weight loss, or locomotor activity. LPS challenge increased (mRNA) Tnfa expression in the hippocampus and PFC of treated mice, whereas etanercept treatment was significantly effective in reversing these effects in the hippocampus only. The effects of etanercept were also associated with a significant reduction in microglial numbers within the hippocampus of these mice following LPS administration.

LPS is a potent and robust model of acute sickness behavior, triggering activation of Toll-like receptors and upregulation of chemokines and cytokines, such as TNF- $\alpha$, 
IL-1 $\beta$, and CXCL1 (Ortega et al, 2011). This leads to acute changes in food intake, fever, hypo-activity, lethargy, and changes in mood in mice (Dantzer et al, 2008), akin to clinical features of depression and anxiety-like behavior (Dantzer et al, 2008; Yirmiya et al, 2001). These features were replicated in this study, with LPS causing weight loss, reduction in locomotor activity as well as cognitive impairment and increased anxiety-like behavior $48 \mathrm{~h}$ postadministration. This corresponds with previous studies that showed that while the peak of peripheral LPS activity, marked by increased cytokine expression, occurs $4-8 \mathrm{~h}$ postadministration, its central effects are delayed. Indeed, it is reported that LPS-related effects on cognition-like behavior manifest as early as $4 \mathrm{~h}$ and are present up to $24 \mathrm{~h}$ postadministration, while depression-like behaviors are evident only from $6 \mathrm{~h}$, up to $24 \mathrm{~h}$ postadministration (Frenois et al, 2007; Noble et al, 2007). In our study, we extended these findings by determining that changes in cognition and anxiety-like behavior were still present $48 \mathrm{~h}$ after LPS administration, although the peak effects of LPS-induced behavioral changes may have been missed due to the delay between LPS treatment and behavioral analyses. The exact mechanisms whereby inflammation induces neurological symptoms are not yet clearly understood (Cavailles et al, 2013; Chandarana et al, 1987; Couch et al, 2013), and in the present study, the anxiety-like effects of LPS appear to be partially dependent on TNF- $\alpha$, as anxiety-like behavior on the EZM could be reduced by central administration of etanercept. Although these effects were significant for head dip and stretch-attend measures only, time spent in open arms showed the same trend across groups, suggesting the effects of LPS and etanercept are consistent across measures. Higher number of mice may have led to all three measures showing significant differences between groups. Additionally, no differences between groups were observed for distance travelled in this test, indicating that the behavior observed was representative of anxiety-like responses and not merely locomotor differences. A role for TNF- $\alpha$ in anxiety-like behavior is supported by studies demonstrating that chronic peripheral administration of TNF- $\alpha$ antagonists reduce levels of depression and anxiety in conditions, including chronic pain (Chen et al, 2013) and RA (Uguz et al, 2009) while genetic knockout of TNF- $\alpha$ lowers anxiety-like behavior in young mice (Camara et al, 2013). The role of TNF- $\alpha$ in regulating emotional responses within the CNS is yet to be determined with suggestions that TNF- $\alpha$ may be involved in the regulation of the serotonin transporter pathway, as well as stimulation of the hypothalamic-pituitary axis, which are involved in generating emotional responses (Bayramgurler et al, 2013; Himmerich et al, 2006; Zhu et al, 2006).

Supporting the present findings, that a single challenge with LPS systemically can increase central TNF- $\alpha$ expression, Bossu et al (2012), showed that a single dose of LPS $(5 \mathrm{mg} / \mathrm{kg})$ was effective in increasing TNF- $\alpha$ expression in the hippocampus and PFC from 7 days after LPS challenge, lasting up to 10 months after challenge and that these changes were associated with cognitive impairments. These results indicate that TNF- $\alpha$, through systemic LPS, can have lasting neuroinflammatory effects in the brain and may lead to cognitive decline. High doses of LPS have also been shown to cause loss of neuronal loss in the frontal cortex along with cognitive impairment (Semmler et al, 2007). LPS challenge has also been linked to decreased parvalbumin reactivity (required for inhibitory neurons) in neurons of the hippocampus without any corresponding change in the frontal regions of rodents (Jenkins et al, 2009). Furthermore, extremely high doses of LPS $(10 \mathrm{mg} / \mathrm{kg}$ and $5 \mathrm{mg} / \mathrm{kg}$ ) were shown to reduce cholinergic innervations (Semmler et al, 2007) and cause degeneration of dopaminergic neurons (Qin et al, 2007). High LPS (5 mg/kg) also increased TNF- $\alpha$ levels in the brain, serum, and liver (Qin et al, 2007). These studies support the current findings of increased (mRNA) Tnfa in the hippocampus and PFC $48 \mathrm{~h}$ after systemic LPS challenge of a relatively lower dose.

In the present study, TNF- $\alpha$ appears to have an important role in mediating the anxiety-like effects induced by LPS as etanercept reduced anxiety-like behaviors. This role of TNF$\alpha$ is supported by previous studies where genetic knockout of TNF- $\alpha$ reduced anxiety-like behavior in young mice (Camara et al, 2013), while central blockade reduced experimental autoimmune encephalitis -induced anxiety in mice (Haji et al, 2012). Additionally, TNF- $\alpha$ antagonists reduced anxiety in chronic pain models in mice after peripheral administration (Chen et al, 2013), and this was supported by decreased anxiety observed in RA patients after antiTNF- $\alpha$ treatment (Uguz et al, 2009). Haji et al (2012) showed that the release of TNF- $\alpha$ from activated microglia altered excitatory transmission within the striatum promotes the development of anxiety-like behavior, whereas etanercept decreased decay time and duration of mini excitatory postsynaptic currents, indicating a reduction in glutamatergic signalling. Other potential mechanisms whereby TNF- $\alpha$ may induce the development of anxietylike behavior include the stimulation of microglia to release glutamate causing neurotoxicity by cell death (Takeuchi et al, 2006) and by silencing cell survival signals (Tamminga, 1998; Venters et al, 2000). Moreover, dysfunction in glutamate signalling has been related to cognitive decline and development of psychotic symptoms (Francis, 2009). Furthermore, it has been suggested that TNF- $\alpha$ modifies the serotonin transporter uptake through p38 mitogen-activated protein kinases and thereby altering emotional behavior, which has been related to anxiety, depression, and other mood disturbances, such as aggression ( Zhu et al, 2006).

Although the present study demonstrated a significant effect of LPS challenge on cognition-like behaviors, independent of changes in locomotor activity in this test, etanercept failed to significantly reverse these deficits. This contrasts previous reports where continuous peripheral TNF- $\alpha$ antagonism in RA patients improved cognition (Chen et al, 2010), suggesting that chronic rather than a single dose administration is needed to achieve such effects. However, it should be noted that Tobinick et al (2006) and Tobinick and Gross, (2008) observed a rapid improvement in cognition in a small cohort of patients with Alzheimer's disease $(\mathrm{AD})$ following a single dose of peri-spinal etanercept and was supported by animal research on $\mathrm{AD}$ models where antagonism of TNF- $\alpha$ by ICV injection had immediate effects on cognition (Medeiros et al, 2007). Additionally, these findings suggest that centrally administered etanercept is more effective in attenuating cognitive impairment in CNS disease models such as $\mathrm{AD}$, which are associated with more substantial cognitive deficits, than in our peripheral inflammatory model induced with a single dose 
of LPS. The lack of significant effects of etanercept in cognition-like behavior in our study may be linked to the time delay between LPS administration and etanercept treatment. As major effects of LPS on cognition have previously only been reported up to $24 \mathrm{~h}$ post-LPS (Noble et al, 2007), the present study may have missed the peak of cognitive dysfunction, although significant deficits were still noted in the LPS-treated mice. Thus measuring the efficacy of etanercept at an earlier time point post-LPS administration may be necessary to observe significant treatment effects on cognition-like behavior. Another alternative for the non-significant effect of etanercept on cognition is the high variation within groups in this test compared with others. Using increased numbers or a more sensitive test of cognition could have helped us see a significant effect of etanercept treatment. Future studies should take this into account as well as timing of drug treatments.

Interestingly, our study failed to observe effects of LPS on astrocytic as opposed to microglia numbers, suggesting differential activation of the two glial cell types, whereby microglia may be more sensitive to peripheral administration of LPS than astrocytes. This may relate to the dose of LPS, as chronic administration (daily for 7 days) generated an astrocyte response in another study, whereas in our study only a single LPS administration was employed (Bian et al, 2013). Another possibility is that there is a temporal difference between the reactivity of the two cell types, with microglia responding more quickly to this insult than astrocytes (Kuhlmann and Guilarte, 2000; Ni et al, 2010). Kuhlmann and Guilarte (2000) also reported that while microglia responded within $48 \mathrm{~h}$ to an insult generated by administration of neurotoxicant trimethyltin, astrocytic response was delayed, seen at 14 days but not $48 \mathrm{~h}$ following injury. This may explain the lack of findings of peripheral LPS administration on astrocytic number in the current study, and future work would need to look at the delayed effects of LPS on astrocytes.

The behavioral deficits seen in this study were associated with increased microglia numbers within the dentate gyrus in LPS-treated mice, $48 \mathrm{~h}$ postadministration, but not in the PFC. It is known that peripherally stimulated monocytes affect the CNS via the BBB and the blood-CSF barrier and express cytokines, such as TNF- $\alpha$ (Ubogu et al, 2006). Indeed, this peripheral-central immune cross-talk is further supported by a study that showed how a single high dose of LPS $(5 \mathrm{mg} / \mathrm{kg}$ ) given IP was effective in increasing cytokine expression, particularly TNF- $\alpha$, in the hippocampus and prefrontal cortex even 7 days after administration (Bossu et al, 2012). Thus, activation of peripheral inflammation can promote central activation and proliferation of microglia and subsequent production of cytokines within the brain itself (Bossu et al, 2012; Qin et al, 2007), providing a potential mechanism to explain the increase in microglia numbers noted within this study only in the hippocampus but not in the PFC. The differences in microglia findings in the hippocampus and the PFC between the present study and previous results by Bossu et al (2012) may be due to the relatively low dose of LPS used in this study. It may be plausible that a higher dose of LPS is required to illicit a change in microglial numbers in the PFC, but the hippocampus is more susceptible to immune activation; however, these speculations will need to be clarified by further studies.
Chronic and acute stressors are known to prime microglia, leading to upregulation of pro-inflammatory cytokines, such as TNF- $\alpha$. Microglia are also believed to be activated by psychological and peripheral immune factors, which can result in behavioral changes (Li et al, 2014). Zhu et al (2014) proposed that, as activated microglia that are known to produce pro-inflammatory cytokines, this may cause neuronal toxicity through free radicals, cytokines, and decreased neurogenesis leading to behavioral impairments, particularly social impairment and prepulse inhibition. Additionally, chronic stress has been shown to increase microglial activation leading to increased uptake of peripheral monocytes perpetuating cognitive and emotional impairment possibly by impairing synaptic connections (Hinwood et al, 2012; Li et al, 2014; Wohleb et al, 2011; Wohleb et al, 2013). Furthermore, high levels of TNF- $\alpha$ promote microglia activation inducing a positive feedback loop causing more activation and proliferation of microglia and TNF- $\alpha$ (Takeuchi et al, 2006). Thereby our study supports that blocking TNF- $\alpha$ action in the brain would be expected to reduce microglia proliferation by stopping this feedback loop. Indeed, studies on traumatic brain injury in rats showed that etanercept administration is effective in reducing microglia numbers within the CNS following injury (Chio et al, 2013).

Overall, this study shows that following LPS-induced behavioral changes and increased microglia in the dentate gyrus, a single dose of etanercept can significantly reduce anxiety-like behavior and microglia numbers $48 \mathrm{~h}$ after peripheral immune challenge. Future work should explore whether a single dose of etanercept administered at the peak of LPS activity (4-8h) will be more effective in reducing inflammation as well as in improving cognition and moodlike behaviors compared with a later point of administration. Furthermore, it would be worth investigating whether prolonged etanercept administration would be more effective than simply increasing the dosage concentration.

\section{FUNDING AND DISCLOSURE}

This study was supported by the National Health and Medical Research Council Australia (APP1003788 to BTB). The funders had no role in study design, data collection and analysis, decision to publish, or preparation of the manuscript. The authors declare no conflict of interest.

\section{ACKNOWLEDGEMENTS}

We thank Jim Manavis and Gaurav Singhal for help with some histological aspects used in this study. We also thank Professor Amanda Page, head, Nerve-Gut Lab School of Medicine, for allowing us to use their ABI 7500 Fast realtime PCR machine and the lab space for conducting the RT-qPCR analysis.

\section{REFERENCES}

Bai YM, Chiou WF, Su TP, Li CT, Chen MH (2013). Proinflammatory cytokine associated with somatic and pain symptoms in depression. J Affect Disord 155: 28-34. 
Bassukas ID, Hyphantis T, Gamvroulia C, Gaitanis G, Mavreas V (2008). Infliximab for patients with plaque psoriasis and severe psychiatric comorbidity. J Eur Acad Dermatol Venereol 22: 257-258.

Baune BT, Camara M-l, Eyre H, Jawahar C, Anscomb H, Koerner H (2012). Tumour necrosis factor alpha mediated mechanisms of cognitive dysfunction. Trans Neurosci 3: 263-277.

Bayramgurler D, Karson A, Ozer C, Utkan T (2013). Effects of longterm etanercept treatment on anxiety- and depression-like neurobehaviors in rats. Physiol Behav 119: 145-148.

Bian Y, Zhao X, Li M, Zeng S, Zhao B (2013). Various roles of astrocytes during recovery from repeated exposure to different doses of lipopolysaccharide. Behav Brain Res 253: 253-261.

Boado RJ, Hui EK, Lu JZ, Zhou QH, Pardridge WM (2010). Selective targeting of a TNFR decoy receptor pharmaceutical to the primate brain as a receptor-specific IgG fusion protein. J Biotechnol 146: 84-91.

Bossu P, Cutuli D, Palladino I, Caporali P, Angelucci F, Laricchiuta D et al (2012). A single intraperitoneal injection of endotoxin in rats induces long-lasting modifications in behavior and brain protein levels of TNF-alpha and IL-18. J Neuroinflammation 9: 101.

Brown RE, Stanford L, Schellinck HM (2000). Developing standardized behavioral tests for knockout and mutant mice. ILAR J 41: 163-174.

Camara ML, Corrigan F, Jaehne EJ, Jawahar MC, Anscomb H, Koerner $\mathrm{H}$ et al (2013). TNF-alpha and its receptors modulate complex behaviours and neurotrophins in transgenic mice. Psychoneuroendocrinology 38: 3102-3114.

Cavailles A, Brinchault-Rabin G, Dixmier A, Goupil F, Gut-Gobert C, Marchand-Adam S et al (2013). Comorbidities of COPD. Eur Respir Rev 22: 454-475.

Chandarana PC, Eals M, Steingart AB, Bellamy N, Allen S (1987). The detection of psychiatric morbidity and associated factors in patients with rheumatoid arthritis. Can J Psychiatry 32: 356-361.

Chen J, Song Y, Yang J, Zhang Y, Zhao P, Zhu XJ et al (2013). The contribution of TNF-alpha in the amygdala to anxiety in mice with persistent inflammatory pain. Neurosci Lett 541: 275-280.

Chen YM, Chen HH, Lan JL, Chen DY (2010). Improvement of cognition, a potential benefit of anti-TNF therapy in elderly patients with rheumatoid arthritis. Joint Bone Spine 77: 366-367.

Chio CC, Chang CH, Wang CC, Cheong CU, Chao CM, Cheng BC et al (2013). Etanercept attenuates traumatic brain injury in rats by reducing early microglial expression of tumor necrosis factoralpha. BMC Neurosci 14: 33.

Choy KH, de Visser Y, Nichols NR, van den Buuse M (2008). Combined neonatal stress and young-adult glucocorticoid stimulation in rats reduce BDNF expression in hippocampus: effects on learning and memory. Hippocampus 18: 655-667.

Couch Y, Anthony DC, Dolgov O, Revischin A, Festoff B, Santos AI et al (2013). Microglial activation, increased TNF and SERT expression in the prefrontal cortex define stress-altered behaviour in mice susceptible to anhedonia. Brain Behav Immun 29: 136-146.

Dantzer R, O'Connor JC, Freund GG, Johnson RW, Kelley KW (2008). From inflammation to sickness and depression: when the immune system subjugates the brain. Nat Rev Neurosci 9: 46-56.

Davidson RJ (2002). Anxiety and affective style: role of prefrontal cortex and amygdala. Biol Psychiatry 51: 68-80.

Dulawa SC, Grandy DK, Low MJ, Paulus MP, Geyer MA (1999). Dopamine D4 receptor-knock-out mice exhibit reduced exploration of novel stimuli. J Neurosci 19: 9550-9556.

Francis PT (2009). Altered glutamate neurotransmission and behaviour in dementia: evidence from studies of memantine. Curr Mol Pharmacol 2: 77-82.

Frenois F, Moreau M, O'Connor J, Lawson M, Micon C, Lestage J et al (2007). Psychoneuroendocrinology 32: 516-531.

Haji N, Mandolesi G, Gentile A, Sacchetti L, Fresegna D, Rossi S et al (2012). TNF-alpha-mediated anxiety in a mouse model of multiple sclerosis. Exp Neurol 237: 296-303.
Himmerich H, Binder EB, Kunzel HE, Schuld A, Lucae S, Uhr M et al (2006). Successful antidepressant therapy restores the disturbed interplay between TNF-alpha system and HPA axis. Biol Psychiatry 60: 882-888.

Hinwood M, Morandini J, Day TA, Walker FR (2012). Evidence that microglia mediate the neurobiological effects of chronic psychological stress on the medial prefrontal cortex. Cereb Cortex 22: 1442-1454.

Hunt L, Emery P (2013). Etanercept in the treatment of rheumatoid arthritis. Expert Opin Biol Ther 13: 1441-1450.

Jenkins TA, Harte MK, Stenson G, Reynolds GP (2009). Neonatal lipopolysaccharide induces pathological changes in parvalbumin immunoreactivity in the hippocampus of the rat. Behav Brain Res 205: 355-359.

Kaster MP, Gadotti VM, Calixto JB, Santos ARS, Rodrigues ALS (2012). Depressive-like behavior induced by tumor necrosis factor- $\alpha$ in mice. Neuropharmacology 62: 419-426.

Kekow J, Moots R, Khandker R, Melin J, Freundlich B, Singh A (2011). Improvements in patient-reported outcomes, symptoms of depression and anxiety, and their association with clinical remission among patients with moderateto-severe active early rheumatoid arthritis. Rheumatology 50: 401-409.

Kerfoot SM, D'Mello C, Nguyen H, Ajuebor MN, Kubes P, Le T et al (2006). TNF-alpha-secreting monocytes are recruited into the brain of cholestatic mice. Hepatology 43: 154-162.

Kuhlmann AC, Guilarte TR (2000). Cellular and subcellular localization of peripheral benzodiazepine receptors after trimethyltin neurotoxicity. J Neurochem 74: 1694-1704.

Li Z, Ma L, Kulesskaya N, Võikar V, Tian L (2014). Microglia are polarized to M1 type in high-anxiety inbred mice in response to lipopolysaccharide challenge. Brain Behav Immun 38: 237-248.

Livak KJ, Schmittgen TD (2001). Analysis of relative gene expression data using real-time quantitative PCR and the 2(Delta Delta C(T)) Method. Methods 25: 402-408.

Medeiros R, Prediger RD, Passos GF, Pandolfo P, Duarte FS, Franco JL et al (2007). Connecting TNF-alpha signaling pathways to iNOS expression in a mouse model of Alzheimer's disease: relevance for the behavioral and synaptic deficits induced by amyloid beta protein. J Neurosci 27: 5394-5404.

Menter A, Augustin M, Signorovitch J, Yu AP, Wu EQ, Gupta SR et al (2010). The effect of adalimumab on reducing depression symptoms in patients with moderate to severe psoriasis: a randomized clinical trial. J Am Acad Dermatol 62: 812-818.

Miller EK, Cohen JD (2001). An integrative theory of prefrontal cortex function. Annu Rev Neurosci 24: 167-202.

Mohler KM, Torrance DS, Smith CA, Goodwin RG, Stremler KE, Fung VP et al (1993). Soluble tumor necrosis factor (TNF) receptors are effective therapeutic agents in lethal endotoxemia and function simultaneously as both TNF carriers and TNF antagonists. J Immunol 151: 1548-1561.

Nadeau S, Rivest S (2000). Role of microglial-derived tumor necrosis factor in mediating CD14 transcription and nuclear factor kappa B activity in the brain during endotoxemia. J Neurosci 20: 3456-3468.

Ni M, Li X, Yin Z, Jiang H, Sidoryk-Wegrzynowicz M, Milatovic D et al (2010). Methylmercury induces acute oxidative stress, altering Nrf2 protein level in primary microglial cells. Toxicol Sci 116: 590-603.

Nilsberth C, Elander L, Hamzic N, Norell M, Lonn J, Engstrom L et al (2009). The role of interleukin-6 in lipopolysaccharideinduced fever by mechanisms independent of prostaglandin E2. Endocrinology 150: 1850-1860.

Noble F, Rubira E, Boulanouar M, Palmier B, Plotkine M, Warnet JM et al (2007). Acute systemic inflammation induces central mitochondrial damage and mnesic deficit in adult Swiss mice. Neurosci Lett 424: 106-110. 
Ortega A, Jadeja V, Zhou H (2011). Postnatal development of lipopolysaccharide-induced inflammatory response in the brain. Inflamm Res 60: 175-185.

Qin L, Wu X, Block ML, Liu Y, Breese GR, Hong JS et al (2007). Systemic LPS causes chronic neuroinflammation and progressive neurodegeneration. Glia 55: 453-462.

Rivest S (2003). Molecular insights on the cerebral innate immune system. Brain Behav Immun 17: 13-19.

Salim S, Asghar M, Taneja M, Hovatta I, Chugh G, Vollert C et al (2011). Potential contribution of oxidative stress and inflammation to anxiety and hypertension. Brain Res 1404: 63-71.

Semmler A, Frisch C, Debeir T, Ramanathan M, Okulla T, Klockgether $\mathrm{T}$ et al (2007). Long-term cognitive impairment, neuronal loss and reduced cortical cholinergic innervation after recovery from sepsis in a rodent model. Exp Neurol 204: 733-740.

Shepherd JK, Grewal SS, Fletcher A, Bill DJ, Dourish CT (1994). Behavioural and pharmacological characterisation of the elevated 'zero-maze' as an animal model of anxiety. Psychopharmacology 116: 56-64.

Sweatt JD (2004). Hippocampal function in cognition. Psychopharmacology 174: 99-110.

Takeuchi H, Jin S, Wang J, Zhang G, Kawanokuchi J, Kuno R et al (2006). Tumor necrosis factor-alpha induces neurotoxicity via glutamate release from hemichannels of activated microglia in an autocrine manner. J Biol Chem 281: 21362-21368.

Tamminga CA (1998). Schizophrenia and glutamatergic transmission. Crit Rev Neurobiol 12: 21-36.

Tobinick E, Gross H, Weinberger A, Cohen H (2006). TNF-alpha modulation for treatment of Alzheimer's disease: a 6-month pilot study. Med Gen Med 8: 25.

Tobinick EL, Gross H (2008). Rapid cognitive improvement in Alzheimer's disease following perispinal etanercept administration. J Neuroinflammation 5: 2.

Tyring S, Gordon KB, Poulin Y, Langley RG, Gottlieb AB, Dunn M et al (2007). Long-term safety and efficacy of $50 \mathrm{mg}$ of etanercept twice weekly in patients with psoriasis. Arch Dermatol 143: 719-726.

Tyring S, Gottlieb A, Papp K, Gordon K, Leonardi C, Wang A et al (2006). Etanercept and clinical outcomes, fatigue, and depres- sion in psoriasis: double-blind placebo-controlled randomised phase III trial. Lancet 367: 29-35.

Ubogu EE, Cossoy MB, Ransohoff RM (2006). The expression and function of chemokines involved in CNS inflammation. Trends Pharmacol Sci 27: 48-55.

Uguz F, Akman C, Kucuksarac S, Tufekci O (2009). Anti-tumor necrosis factor-alpha therapy is associated with less frequent mood and anxiety disorders in patients with rheumatoid arthritis. Psychiatry Clin Neurosci 63: 50-55.

Venters HD, Dantzer R, Kelley KW (2000). A new concept in neurodegeneration: TNFalpha is a silencer of survival signals. Trends Neurosci 23: 175-180.

Wohleb ES, Hanke ML, Corona AW, Powell ND, Stiner LM, Bailey MT et al (2011). beta-Adrenergic receptor antagonism prevents anxiety-like behavior and microglial reactivity induced by repeated social defeat. J Neurosci 31: 6277-6288.

Wohleb ES, Powell ND, Godbout JP, Sheridan JF (2013). Stressinduced recruitment of bone marrow-derived monocytes to the brain promotes anxiety-like behavior. J Neurosci 33: 13820 13833.

Yirmiya R, Pollak Y, Barak O, Avitsur R, Ovadia H, Bette M et al (2001). Effects of antidepressant drugs on the behavioral and physiological responses to lipopolysaccharide (LPS) in rodents. Neuropsychopharmacology 24: 531-544.

Zhang X, Wang J, Qian W, Zhao J, Sun L, Qian Y et al (2014). Dexmedetomidine inhibits tumor necrosis factor-alpha and interleukin 6 in lipopolysaccharide-stimulated astrocytes by suppression of c-Jun $\mathrm{N}$-terminal kinases. Inflammation 37: 942-949.

Zhu CB, Blakely RD, Hewlett WA (2006). The proinflammatory cytokines interleukin-1beta and tumor necrosis factor-alpha activate serotonin transporters. Neuropsychopharmacology 31: 2121-2131.

Zhu F, Zhang L, Ding YQ, Zhao J, Zheng Y (2014). Neonatal intrahippocampal injection of lipopolysaccharide induces deficits in social behavior and prepulse inhibition and microglial activation in rats: Implication for a new schizophrenia animal model. Brain Behav Immun 38: 166-174.

Supplementary Information accompanies the paper on the Neuropsychopharmacology website (http://www.nature.com/npp) 